\title{
Angelica Sinensis Extract : A Potential Drug to Enhance Osseointegration of Dental Implants in Osteoporosis Patients
}

\author{
Fei Gao ${ }^{1}$ and Shimao Yang ${ }^{2 *}$ \\ ${ }^{1}$ Department of Anesthesiology, PR China \\ ${ }^{2}$ Department of Oral and Maxillofacial Surgery, PR China
}

*Corresponding author: Shimao Yang, Department of Oral and Maxillofacial Surgery, Jinan Stomatology Hospital, Jinan, PR China

\section{ARTICLE INFO}

Received: 峟 December 02, 2019

Published: December 05, 2019

Citation: Fei Gao, Shimao Yang. Angelica Sinensis Extract: A Potential Drug to Enhance Osseointegration of Dental Implants in Osteoporosis Patients. Biomed J Sci \& Tech Res 23(4)-2019. BJSTR. MS.ID.003933.

Keywords: Angelica Sinensis; Postmenopausal Osteoporosis; Oestrogen; Dental Implant; Osseointegration
ABSTRACT

Background: Postmenopausal osteoporosis is a common skeletal disorder disease because of estrogen deficiency, characterized by increased bone turnover and reductions in bone mineral density. This disorder is a potential risk factor for dental implant surgery because it could increase the risk of oral infectious disease and decrease osseointegration around implants.

Presentation of the Hypothesis: Angelica sinensis root is one of the herbs most commonly used in China. Angelica sinensis extract (AS extract) has been reported to possess neuroprotective, anti-oxidant, hepatoprotective, anti-osteoarthritis, anti-cancer effects. It has also been utilized as a valuable remedy for anemia, menstrual irregularities, and constipation. Recent studies reveal that the AS extract has anti-osteoporotic effects on ovariectomized rats and could promote the proliferation of human bone cells. We therefore hypothesize that systemically or locally used AS extract could improve osseointegration of dental implants in osteoporosis patients.

Implications of the Hypothesis: Our hypothesis could contribute to provide an option to enhance success ratio of dental implants in postmenopausal osteoporosis by the replenishment of AS extract.

Abbreviations: TCM: Traditional Chinese Medicine, AS: Angelica Sinensis, ALP: Alkalinephosphatase, VOAS: Volatile Oils of Angelica Sinensis

\section{Introduction}

Postmenopausal osteoporosis is a common skeletal disorder disease which has become one of the most important public health problems in our ageing society. It is mainly caused by estrogen deficiency, while the lack of estrogen after menopause is associated with increased osteoblast (bone-forming cell) apoptosis [1]. These factors can lead to the increase of both osteoclastogenesis and osteoclast activity, eventually causing decreased bone mass and increased risk for osteoporosis [2]. Furthermore, genetic and hereditary factors, hormonal status, dietary habits as well as lifestyle may also involve the development of postmenopausal osteoporosis [3-5]. Some reports had showed that osteoporosis could impact the osteointegration owing to the skeletal disorder and changes of the bone remodeling, which was associated within creased bone turnover and reductions in bone mineral density [68]. Recent study indicates that inflammation also plays a critical role in bone remodeling and in pathogenesis of osteoporosis $[9,10]$.

It is well known that the immune and skeletal systems interact and affect each other [11-14]. Recent years, the most frequently studied topics concern osteoporosis is focus on the the immune system, pro-inflammatory cytokines with their receptors and immunological mechanisms regulating bone metabolism. Oestrogen plays an important role in the regulation of immune function, and its receptors have been identified on monocytes, $\mathrm{T}$ and $\mathrm{B}$ lymphocytes [15]. Oestrogen deficiency could result in a marked increase in pro- 
inflammatory cytokines, whereas the level of bone-forming factors is decreased $[15,16]$. Therefore, oestrogen deficiency may provide a more susceptible environment for bacteria and lead to the increase the risk of oral infectious disease, which may have a relationship with the imbalanced bone status of osteoporosis [17]. These elements make osteoporosis patients prone to peri-implantitis, which could be a potential risk factor for implants in dental surgery because it could decrease bone formation around implants and then affect osseointegration of dental implants.

Many previous studies have proved that the rate of osseointegration around dental implants is significantly reduced under osteoporotic conditions compared to the normal conditions $[18,19]$. Therefore, how to avoid implant loss and enhance success ratio of dental implants in postmenopausal osteoporosis patients is always a clinical focus. Angelica Sinensis (AS) root is one of the herbs most widely used in China. AS extract has been reported to possess neuroprotective, anti-oxidant, hepatoprotective, anti-osteoarthritis, anti-cancer effects [20-24]. It has also been utilized in Traditional Chinese Medicine (TCM) as a valuable remedy for anemia, menstrual irregularities, and constipation [25-28]. The diverse biological activities of AS extract mainly depend on the active compounds of phthalides, organic acids, polysaccharides, and flavones [29]. Recently, it has been recognized that the AS extract has anti-osteoporotic effects on ovariectomized rats [30]. Moreover, it could promote the proliferation and differentiation of human bone cells [31]. It also has been suggested that AS extract could inhibit RANKL-mediated osteoclast differentiation in bone marrow macrophages in vitro, indicating that it may serve as a useful drug in the prevention of bone loss [32].

\section{Presentation of The Hypothesis}

Our hypothesis is that systemically administration or locally used AS extract could enhance osseointegration of dental implants in postmenopausal osteoporosis patients. Then, it could provide an option to improve success ratio of dental implants in postmenopausal osteoporosis patients through the replenishment of AS extract. Angelica sinensis has been always used in traditional prescriptions for bone and tendon injuries. Hu XM reported that Angelica sinensis was one of the main ingredients in the Encyclopedia of Esoteric Prescriptions in TCM of fracture prescriptions [33]. It has shown that these prescriptions had significant effect in reducing the time needed for the injured bones to heal. AS extract was found to directly stimulate the proliferation of human bone cells, and improve the Alkalinephosphatase (ALP) activity, type I collagen synthesis of OPC-1 at dose-dependent manner [31]. It is possible that the extract of Angelica sinensis promoted mineralization of the organicmatrix, and then speeding up bone formation.

Results of the recent research showed that AS extract could prevent OVX-induced bone loss with efficacy comparable to that of estrogen. It significantly decreased the bone (femur) mineral density (BMD) loss, and the levels of bone turnover markers including serum ALP, Osteocalcin (OC), and Collagen type I C-telopeptide (CTx) compared to the OVX control group without influencing estrogen level were reduced markly [30]. Moreover, the preliminary study performed by our team recently in vitro indicated that AS extract could promote the mineralization as well as ALP activity of MC3T3 in a dose-dependent (Figure1). These results indicate that AS extract could be an effective natural alternative for the treatment of postmenopausal osteoporosis (Figures $1 \& 2$ ).
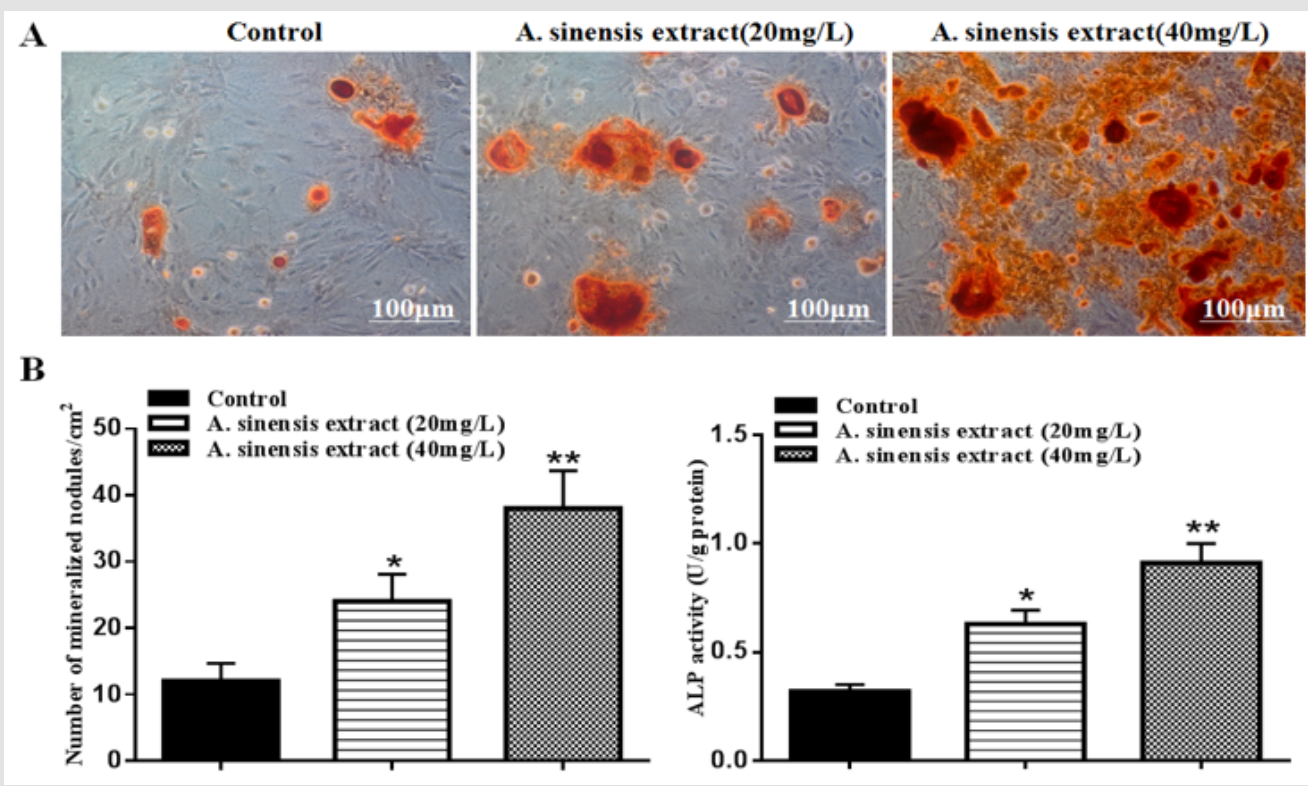

Figure 1: Alizarin red staining of MC3T3 cells. A: Microscopy observation of alizarin red-positive nodule stained MC3T3 cells inosteogenic medium with or without ASextract $(20$ or $40 \mathrm{mg} / \mathrm{L})$ for 2 weeks. B: Quantitation of the density of alizarin redpositive nodules formed and ALP assay of cells in different groups. Data from 3 cultures with triplicates were expressed as mean $\pm S D$, error bars in the figure were indicated for SD; ${ }^{*} p<0.05$ and ${ }^{* *} p<0.01$ vs. control. 


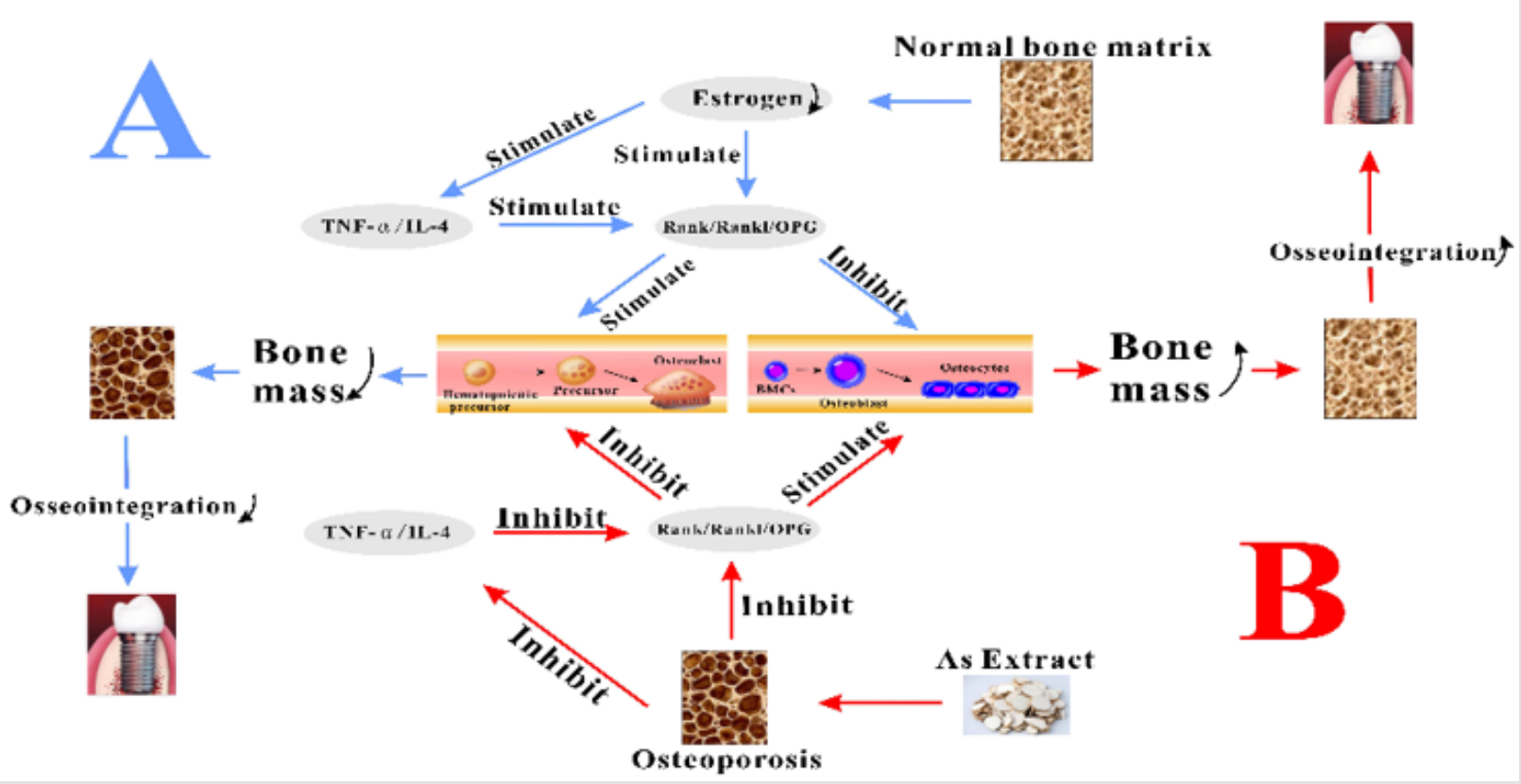

Figure 2: Effect of estrogen and AS Extract on bone mass A: Indicated by the blue rows show the possible mechanisms of estrogen causing bone loss. B: Indicated by the red rows show the possible mechanisms of As Extract increasing bone mass.

AS extract has been demonstrated the inhibitory effects on RANKL-mediated osteoclast differentiation from macrophages and bone resorption in vitro, and it also reduced the RANKL-induced expression of osteoclastic marker genes. Furthermore, AS extract weakened the activation of RANKL-induced signaling pathway including ERK, p38, JNK, NF- $\kappa B$, AP-1and NFATc1 [32]. This observation suggests that AS extract may as a potential drug in the therapy for disorders associated with bone loss. Angelica sinensis has been used alone, or in combination with other traditional Chinese herbs, to treat various inflammatory diseases [34-36]. AS extract was found to have anti-inflammatory effects and activates the Nrf2 pathway, which protects against oxidative stress [37]. The aqueous extract of Angelica sinensis can inhibit wear debris particles-induced osteolysis through its ability of inhibiting TNF- $\alpha$ and IL-1 $\beta$ release by macrophages, providing a new possible way to prevent and treat aseptic loosening after total joint replacement [38].

In addition, Zhang WQ et al showed that the other form of Angelica sinensis, Volatile Oils of Angelica Sinensis (VOAS), could intervened in the metabolic process of inflammation by altering histidine metabolism, tryptophan metabolism, arachidonic acid metabolism, steroid hormone biosynthesis, fatty acid metabolism and energy metabolism. Metabonomics was used to reflect an organism's physiological and metabolic state comprehensively, and the VOAS was regarded as a potentially powerful tool that reveals anti-acute-inflammatory mechanism [39]. Therefore, the antiinflammatory properties of AS extract may be a major component of its beneficial effects on promotion of osseointegration of dental implants in postmenopausal osteoporosis.

\section{Implications of The Hypothesis}

Postmenopausal osteoporosis poses a significant threat to millions of postmenopausal women. The lack of estrogen after menopause could activate Rank/Rankl/OPG signaling or TNF- $\alpha$ / IL-1 signaling which directly or indirectly stimulates osteocalst differentiation while inhibit osteoblast differentiation, and then resulting in bone loss as well as microarchitectural deterioration including bone fragility and an increased risk of fracture. Recent investigation has demonstrated that AS extract could prevent bone loss in OVX rats [30].AS extract could directly inhibit Rank/Rankl/ OPG signaling and further prevent osteoclast differentiation [32]. Furthermore, AS extract also could decrease the OVX-induced serum TNF- $\alpha$ and IL-1 levels and indirectly inhibit Rank/Rankl/ OPG signaling, then leading to osteoclast differentiation reduced while osteoblast differentiation increased (Figure 2). Accordingly, to provide an option to improve success ratio of dental implants in osteoporosis patients, we hypothesize that systemically or locally used AS extract could enhance osseointegration of dental implants in postmenopausal osteoporosis patients.

\section{Conclusion}

This hypothesis may contribute to provide an approach to decrease the failure ratio of dental implants in postmenopausal osteoporosis. However, further studies must be performed to prove our hypothesis.

\section{Acknowledgement}

None. 


\section{Funding}

This study was supported by Grants from the Open Project of Shandong Provincial Key Laboratory of Oral Tissue Regeneration: SDKQ201905.

\section{Authors' Contributions}

Shimao Yang conceived the idea and formulated the main lines of the theory. Fei Gao performed data collection wrote the paper. All authors read and approved the final manuscript.

\section{Competing Interests}

The author declares that they have no competing interests.

\section{Consent for Publication}

Not applicable. Ethics approval and consent to participate.

\section{References}

1. Lerner UH (2006) Bone remodeling in post-menopausal osteoporosis. J Dent Res 85: 584-595.

2. Pacifici R (1996) Estrogen, cytokines, and pathogenesis of postmenopausal osteoporosis. J Bone Miner Res 11: 1043-1051.

3. Ralston SH, Uitterlinden A (2010) Genetics of osteoporosis.Endoc Rev 31: 629-662.

4. Warriner AH, Saag KG (2013) Osteoporosis diagnosis and medical treatment. OrthopClin North Am 44: 125-135.

5. Tatsuno I, Terano T, Nakamura M, Suzuki K, Kubota K, et al. (2013) Lifestyle and osteoporosis in middle-aged and elderly women: Chiba bone survey. Endocr J 60: 643-650.

6. Lugero GG, De Falco Caparbo V, Guzzo ML, KonigJr B, Jorgetti V (2000) Histomorphometric evaluation of titanium implants in osteoporotic rabbits. Implant Dent 9: 303-309.

7. Kurth AH, Eberhardt C, Muller S, Steinacker M, Schwarz M (2005) The bisphosphonate ibandronate improves implant integration in osteopenicovariectomized rats. Bone 37: 204-210.

8. Saito T, Kin Y, Koshino T (2002) Osteogenic response of hydroxyapatite cement implanted into the femur of rats with experimentally induced osteoporosis. Biomaterials 23: 2711-2716.

9. Mundy GR (2007) Osteoporosis and inflammation. Nutr Rev 65: 147151.

10. Braun T, Schett G (2012) Pathways for bone loss in inflammatory disease. CurrOsteoporos Rep 10: 101-108.

11. Walsh MC, Kim N, Kadono Y, Rho J, Lee SY, et al. (2006) Osteoimmunology: interplay between the immune system and bone metabolism. Annu Rev Immunol 24: 33-63.

12. Lorenzo J, Horowitz M, Choi Y (2008) Osteoimmunology: interactions of the bone and immune system. Endocr Rev 29: 403-440.

13. Mori G, D’Amelio P, Faccio R, Brunetti G (2013) The interplay between the bone and the immune system.Clin Dev Immunol 720504.

14. Danks L, Takayanagi H (2013) Immunology and bone. J Biochem 154: 29-39.

15. Clowes JA, Riggs BL, Khosla S (2005) The role of the immune system in the pathophysiology of osteoporosis. Immunological Rev 208: 207-227.

16. Marco F, Milena F, Gianluca G, Vittoria O (2005) Peri-implant osteogenesis in health and osteoporosis. Micron 36: 630-644.

17. Brennan Calanan, Genco RM, Wilding RJ, Hovey GE, Trevisan M, et al. (2008) Osteoporosis and oral infection: independent risk factors for oral bone loss. J Dent Res 87: 323-327.
18. Lugero GG, De Falco Caparbo V, Guzzo ML, KonigJr B, Jorgetti V (2000) Histomorphometric evaluation of titanium implants in osteoporotic rabbits. Implant Dent 9: 303-309.

19. Kurth AH, Eberhardt C, Muller S, Steinacker M, Schwarz M (2005) The bisphosphonate ibandronate improves implant integration in osteopenic ovariectomized rats. Bone 37: 204-210.

20. Ye YN, Liu ES, Li Y, So HL, Cho CC, et al. (2001) Protective effect of polysaccharides-enriched fraction from angelica sinensis on hepatic injury. Life Sci 69: 637-646.

21. Huang SH, Lin CM, Chiang BH (2008) Protective effects of angelica sinensis extract on amyloid beta-peptide-induced neurotoxicity. Phytomed. Int J Phytother Phytopharmacol 15: 710-721.

22. Wu SJ, Ng LT, Lin CC (2004) Antioxidant activities of some common ingredients of traditional chinese medicine, angelica sinensis, lyciumbarbarum and poriacocos. Phytother Res 18: 1008-12.

23. Qin J, Liu YS, Liu J, Li J, Tan Y, et al. (2013) Effect of angelica sinensis polysaccharides on osteoarthritis in vivo and in vitro: A possible mechanism to promote proteoglycans synthesis. Evid Based Complement Altern Med pp. 794761.

24. Lai JN, Wu CT, Wang JD (2012) Prescription pattern of chinese herbal products for breast cancer in taiwan: A population-based study. Evid Based Complement Altern Med pp. 891893.

25. Yim TK, Wu WK, Pak WF, Mak DH, Liang SM, et al. (2000) Myocardial protection against ischaemia-reperfusion injury by a polygonum multiflorum extract supplemented "Dang-Gui decoction for enriching blood", a compound formulation, ex vivo. Phytother Res 14: 195-199.

26. Ji SG, Chai YF, Wu YT, Yin XP, Liang DS, et al. (1999) Determination of ferulic acid in Angelica sinensis and Chuanxiong by capillary zone electrophoresis. Biomed Chromatogr 13: 333-334.

27. Ye YN, Liu ES, Li Y, So HL, Cho CC, et al. (2001) Protective effect of polysaccharides-enriched fraction from Angelica sinensis on hepatic injury. Life Sci 69: 637-646.

28. Liu J, Burdette JE, Xu H, Gu C, van Breemen RB, et al. (2001) Evaluation of estrogenic activity of plant extracts for the potential treatment of menopausal symptoms. J Agric Food Chem 49: 2472-2479.

29. Chen XP, Li W, Xiao XF, Zhang LL, Liu CX (2013) Phytochemical and pharmacological studies on radix angelica sinensis. Chin J Nat Med 11: 577-587.

30. Lim DW, Kim YT (2014) Anti-Osteoporotic Effects of Angelica sinensis (Oliv.) Diels Extract on Ovariectomized Rats and Its Oral Toxicity in Rats. Nutrient 16: 4362-4372.

31. Yang Q, Populo SM, Zhang J, Yang G, Kodama H (2002) Effect of Angelica sinensis on the proliferation of human bone cells. Clin Chim Acta 324: 89-97.

32. Kong L, Zhao Q, Wang X, Zhu J, Hao D (2014) Angelica sinensis extract inhibits RANKL-mediated osteoclastogenesis by down-regulated the expression ofNFATc1 in mouse bone marrow cells. BMC Complement Altern Med 12: 481.

33. Hu XM (1992) Encyclopedia of esoteric prescriptions in traditional Chinese medicine. Surgery/Bone Injury 4: 295-368.

34. Chen MP, Yang SH, Chou CH, Yang KC, Wu CC, et al. (2010) The chondroprotective effects of ferulic acid on hydrogen peroxidestimulated chondrocytes: Inhibition of hydrogen peroxide-induced proinflammatory cytokines and metalloproteinase gene expression at the mRNA level. Inflamm Res 59: 587-95.

35. Jung SM, Schumacher HR, Kim H, Kim M, Lee SH, et al. (2007) Reduction of urate crystal-induced inflammation by root extracts from traditional oriental medicinal plants: Elevation of prostaglandin D2 levels. Arthritis Res There 9: R64.

36. Lin Z, Zhu D, Yan Y, Yu B (2008) Herbal formula FBD extracts prevented brain injury and inflammation induced by cerebral ischemia-reperfusion. J Ethnopharmacol 118: 140-147. 
37. Saw CL, Wu Q, Su ZY, Wang H, Yang Y, et al. (2013) Effects of natural phytochemicals in Angelica sinensis (Danggui) on Nrf2-mediated gene expression of phase II drug metabolizing enzymes and antiinflammation. Biopharm Drug Dispos 34: 303-311.

38. Yang C, Niu S, Yu L, Zhu S, Zhu J, et al. (2012) The aqueous extract of Angelica sinensis, a popular Chinese herb, inhibits wear debris-induced inflammatory osteolysis in mice. J Surg Res 76: 476-483.

\section{ISSN: 2574-1241}

DOI: $10.26717 /$ BJSTR.2019.23.003933

Shimao Yang. Biomed J Sci \& Tech Res

(C) This work is licensed under Creative

Submission Link: https://biomedres.us/submit-manuscript.php
39. Zhang WQ Hua YL, Zhang M, Ji P, Li JX, et al, (2015) Metabonomic analysis of the anti-inflammatory effects of volatile oils of Angelica sinensis on rat model of acute inflammation. Biomed Chromatogr 29: 902-910.

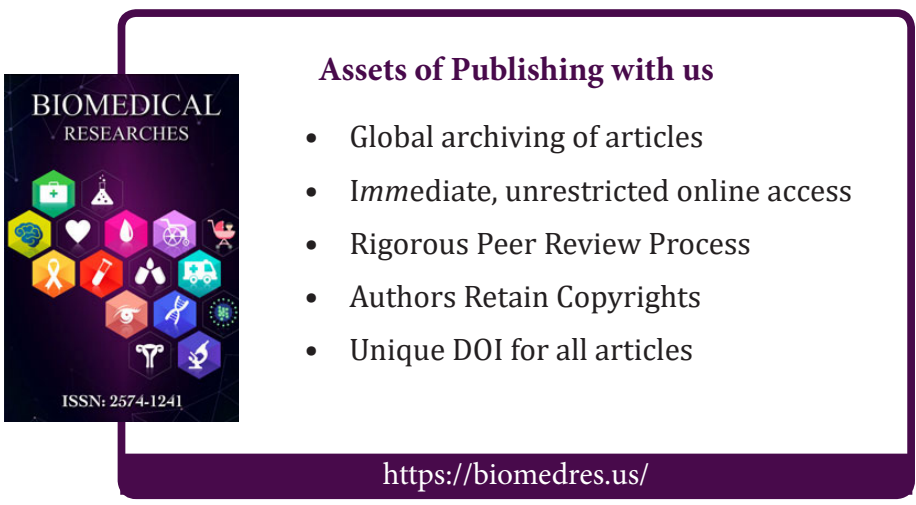

